\title{
Global Finite-Time Stabilization for a Class of Uncertain High-Order Nonlinear Systems
}

\author{
Jian Wang, ${ }^{1}$ Jing Xie, ${ }^{2}$ and Fangzheng Gao ${ }^{1}$ \\ ${ }^{1}$ School of Mathematics and Statistics, Anyang Normal University, Anyang 455002, China \\ ${ }^{2}$ School of Mathematics and Physics, Anyang Institute of Technology, Anyang 455000, China \\ Correspondence should be addressed to Fangzheng Gao; gaofz@126.com
}

Received 10 February 2014; Accepted 27 March 2014; Published 22 April 2014

Academic Editor: Douglas R. Anderson

Copyright (c) 2014 Jian Wang et al. This is an open access article distributed under the Creative Commons Attribution License, which permits unrestricted use, distribution, and reproduction in any medium, provided the original work is properly cited.

\begin{abstract}
This paper addresses the problem of global finite-time stabilization by state feedback for a class of high-order nonlinear systems under weaker condition. By using the methods of adding a power integrator, a continuous state feedback controller is successfully constructed to guarantee the global finite-time stability of the resulting closed-loop system. A simulation example is provided to illustrate the effectiveness of the proposed approach.
\end{abstract}

\section{Introduction}

In this paper, we consider the following high-order nonlinear systems:

$$
\begin{gathered}
\dot{x}_{i}=x_{i+1}^{p_{i}}+f_{i}(t, x, u), \quad i=1, \ldots, n-1, \\
\dot{x}_{n}=u^{p_{n}}+f_{n}(t, x, u),
\end{gathered}
$$

where $x=\left(x_{1}, \ldots, x_{n}\right)^{T} \in R^{n}, u \in R$ are the system state and input, respectively; $p_{i} \in R_{\text {odd }}^{\geq 1}:=\{p / q \mid p$ and $q$ are positive odd integers, and $p \geq q\}$ are said to be the high orders of the system; $f_{i}: R^{+} \times R^{n} \times R \rightarrow R, i=1, \ldots, n$, are unknown continuous functions of all the states and the control input.

The importance for studying such system is exemplified in [1], where state feedback controller was used to stabilize the underactuated, weakly coupled, unstable mechanical system. Since Jacobian linearization of system (1) at the origin is neither controllable nor feedback linearizable for the case of $p>1$, the traditional design tools including feedback linearization or backstepping are hardly applicable to the system (1). Mainly thanks to the adding a power integrator method, a series of stabilizing results have been achieved over the last decades; for example, one can see [2-10] and the references therein. However, it should be mentioned that the aforementioned works only consider the feedback stabilizer that makes the trajectories of the systems converge to the equilibrium as the time goes to infinity.

Compared to the asymptotic stabilization, the finite-time stabilization, which renders the trajectories of the closed-loop systems convergent to the origin in a finite time, has many advantages such as fast response, high tracking precision, and disturbance-rejection properties [11]. Hence it is more meaningful to investigate the finite-time stabilization problem than the classical asymptotical stabilization. In recent years, the finite-time stabilization of system (1) has been studied fairly extensively with various restrictions on the integrator powers and the system nonlinearities [12-21]. In particular, [22] solved the finite-time stabilization problem under the condition that $f_{i}$ satisfies

$$
\left|f_{i}(t, x, u)\right| \leq M \sum_{j=1}^{i}\left|x_{j}\right|^{\left(r_{i}+\omega\right) / r_{j}}
$$

with constants $M>0$ and $\omega \in\left(-2 /(2 n+1) p_{1} \cdots p_{n-1}, 0\right)$. However, from both practical and theoretical points of view, it is somewhat restrictive to require system (1) to satisfy such restriction. Therefore, the following interesting problem is proposed: is it possible to relax the nonlinear growth condition? Under the weaker condition, can a finite-time stabilizing controller be designed? 
In this paper, by necessarily modifying the method of adding a power integrator and by successfully overcoming some essential difficulties such as the weaker assumption on the system growth, the appearance of the sign function, and the construction of a continuously differentiable, positivedefinite, and proper Lyapunov function, we will focus on solving the above problem.

Notations. Throughout this paper, the following notations are adopted. $R^{+}$denotes the set of all nonnegative real numbers and $R^{n}$ denotes the real $n$-dimensional space. For any vector $x=\left(x_{1}, \ldots, x_{n}\right)^{T} \in R^{n}$ denote $\bar{x}_{i}=\left(x_{1}, \ldots, x_{i}\right)^{T} \in R^{i}$, $i=1, \ldots, n,\|x\|=\left(\sum_{i=1}^{n} x_{i}^{2}\right)^{1 / 2} . K$ denotes the set of all functions: $R^{+} \rightarrow R^{+}$, which are continuous, strictly increasing, and vanishing at zero; $K_{\infty}$ denotes the set of all functions which are of class $K$ and unbounded. A sign function $\operatorname{sign}(x)$ is defined as follows: $\operatorname{sign}(x)=1$, if $x>0$; $\operatorname{sign}(x)=0$, if $x=0$; and $\operatorname{sign}(x)=-1$, if $x<0$. Besides, the arguments of the functions (or the functionals) will be omitted or simplified, whenever no confusion can arise from the context. For instance, we sometimes denote a function $f(x(t))$ by simply $f(x), f(\cdot)$, or $f$.

\section{Problem Statement and Preliminaries}

The objective of this paper is to develop a recursive design method for globally finite-time stabilizing system (1) via state feedback under the following assumption.

Assumption 1. For $i=1, \ldots, n$, there are smooth functions $\varphi_{i}\left(\bar{x}_{i}\right) \geq 0$ and constant $\omega \in\left(-1 / \sum_{l=1}^{n} p_{1} \cdots p_{l-1}, 0\right)$ such that

$$
\left|f_{i}(t, x, u)\right| \leq \varphi_{i}\left(\bar{x}_{i}\right) \sum_{j=1}^{i}\left|x_{j}\right|^{\left(r_{i}+\omega\right) / r_{j}}
$$

where $r_{i}$ 's are defined as

$$
r_{1}=1, \quad r_{i+1}=\frac{r_{i}+\omega}{p_{i}}, \quad i=1, \ldots, n .
$$

Remark 2. It is worth pointing out that Assumption 1, which gives the nonlinear growth condition on the system drift terms, encompasses the assumptions in the closely related works $[14,21,22]$. To clearly show this, we would like to make the following comparisons to reveal the relationship between Assumption 1 and the counterparts in [14, 21, 22]; that is, Assumption 1 includes those as special cases.

(i) In $[14,21]$, the system nonlinearities $f_{i}$ 's are required to satisfy

$$
\left|f_{i}(t, x, u)\right| \leq \gamma_{i}\left(\bar{x}_{i}\right)\left(\left|x_{1}\right|+\cdots+\left|x_{i}\right|\right)
$$

where $\gamma_{i}\left(\bar{x}_{i}\right) \geq 0, i=1, \ldots, n$, are $C^{1}$ functions. By (4), we get that $r_{i}=\left(1 / p_{1} \cdots p_{i-1}\right)+\sum_{l=1}^{i-1}\left(\omega / p_{l} \cdots p_{i-1}\right)$. Furthermore, from $\omega \in\left(-1 / \sum_{l=1}^{n} p_{1} \cdots p_{l-1}, 0\right)$, we have $\left(r_{i}+\omega\right) / r_{j}<1$, $i=1, \ldots, n$. It means that

$$
\begin{aligned}
\left|f_{i}(t, x, u)\right| \leq & \gamma_{i}\left(\bar{x}_{i}\right)\left(\left|x_{1}\right|+\cdots+\left|x_{i}\right|\right) \\
\leq \gamma_{i}\left(\bar{x}_{i}\right)( & \left|x_{1}\right|^{\left(r_{i}+\omega\right) / r_{1}}\left|x_{1}\right|^{1-\left(r_{i}+\omega\right) / r_{1}} \\
& \left.+\cdots+\left|x_{i}\right|^{\left(r_{i}+\omega\right) / r_{i}}\left|x_{i}\right|^{1-\left(r_{i}+\omega\right) / r_{i}}\right) .
\end{aligned}
$$

Letting $\varphi_{i}\left(\bar{x}_{i}\right)$ be smooth functions and satisfying $\varphi_{i}\left(\bar{x}_{i}\right) \geq$ $\max \left\{\gamma_{i}\left|x_{1}\right|^{1-\left(r_{i}+\omega\right) / r_{1}}, \ldots, \gamma_{i}\left|x_{i}\right|^{1-\left(r_{i}+\omega\right) / r_{i}}\right\}$, we have

$$
\left|f_{i}(t, x, u)\right| \leq \varphi_{i}\left(\bar{x}_{i}\right)\left(\left|x_{1}\right|^{\left(r_{i}+\omega\right) / r_{1}}+\cdots+\left|x_{i}\right|^{\left(r_{i}+\omega\right) / r_{i}}\right) ;
$$

from this we can see that the main assumption (4) in [14, 21] is a special case of Assumption 1 above.

(ii) In [22], the system nonlinearities $f_{i}$ 's are required to satisfy

$$
\left|f_{i}(t, x, u)\right| \leq M \sum_{j=1}^{i}\left|x_{j}\right|^{\left(r_{i}+\omega\right) / r_{j}}
$$

with constants $M>0$ and $\omega \in\left(-2 /(2 n+1) p_{1} \cdots p_{n-1}, 0\right)$. Obviously, when $\varphi_{i}\left(\bar{x}_{i}\right)=M$, inequality (4) degenerates to inequality (8). Moreover, the value range of $\omega$ in (4) is larger than that in (8). Thus, the main assumption (5) in [22] is a special case of Assumption 1 above.

In the remainder of this section, we present the following lemmas which play an important role in the design process.

Lemma 3 (see [11]). Consider the nonlinear system

$$
\dot{x}=f(x) \text { with } f(0)=0, \quad x \in R^{n},
$$

where $f: U_{0} \rightarrow R^{n}$ is continuous with respect to $x$ on an open neighborhood $U_{0}$ of the origin $x=0$. Suppose that there is a $C^{1}$ function $V(x)$ defined in a neighborhood $\widehat{U} \in R^{n}$ of the origin, real numbers $c>0$ and $0<\alpha<1$, such that

(i) $V(x)$ is positive definite on $\widehat{U}$;

(ii) $\dot{V}(x)+c V^{\alpha}(x) \leq 0, \forall x \in \widehat{U}$.

Then, the origin of (9) is finite-time stable with

$$
T \leq \frac{V^{1-\alpha}(x(0))}{c(1-\alpha)}
$$

for initial condition $x(0)$ in some open neighborhood $U \in \widehat{U}$ of the origin. If $U=R^{n}$ and $V(x)$ is radially unbounded (i.e., $V(x) \rightarrow+\infty$ as $x \rightarrow+\infty$ ), the origin of system (9) is globally finite-time stable.

Lemma 4 (see [6]). For $x, y \in R$, and $p \geq 1$ being a constant, the following inequalities hold:

$$
\begin{gathered}
|x+y|^{p} \leq 2^{p-1}\left|x^{p}+y^{p}\right| . \\
(|x|+|y|)^{1 / p} \leq|x|^{1 / p}+|y|^{1 / p} \leq 2^{(p-1) / p}(|x|+|y|)^{1 / p}
\end{gathered}
$$


If $p \geq 1$ is odd, then

$$
\begin{gathered}
|x-y|^{p} \leq 2^{p-1}\left|x^{p}-y^{p}\right| \\
\left|x^{1 / p}-y^{1 / p}\right| \leq 2^{(p-1) / p}|x-y|^{1 / p} .
\end{gathered}
$$

Lemma 5 (see [10]). If $p=a / b \in R_{\text {odd }}^{\geq 1}$ with $b \geq 1$, then for any $x, y \in R$

$$
\left|x^{p}-y^{p}\right| \leq\left. 2^{1-1 / b}|\operatorname{sgn}(x)| x\right|^{a}-\left.\operatorname{sgn}(y)|y|^{a}\right|^{1 / b} .
$$

Lemma 6 (see [23]). Let $x, y$ be real variables; then for any positive real numbers $a, m$, and $n$, one has

$$
\begin{aligned}
a|x|^{m}|y|^{n} \leq & b|x|^{m+n}+\frac{n}{m+n}\left(\frac{m+n}{m}\right)^{-m / n} \\
& \times a^{(m+n) / n} b^{-m / n}|y|^{m+n}
\end{aligned}
$$

where $b>0$ is any real number.

\section{Finite-Time Control Design}

In this section, we will construct a continuous state feedback controller by applying the method of adding a power integrator. For simplicity, we denote $\operatorname{sgn}(x)|x|^{a} \triangleq[x]^{a}$ for any $a \in R^{+}$ and $x \in R$.

Step 1. Let $\xi_{1}=\left[x_{1}\right]$ and choose $V_{1}=W_{1}=\int_{0}^{x_{1}} s^{2-r_{2} p_{1}} d s$. Using (1) and Assumption 1, we have

$$
\dot{V}_{1}=x_{1}^{2-r_{2} p_{1}} x_{2}^{p_{1}}+x_{1}^{2-r_{2} p_{1}} f_{1} \leq x_{1}^{2-r_{2} p_{1}} x_{2}^{p_{1}}+x_{1}^{2} \varphi_{1} .
$$

Obviously, the first virtual controller

$$
x_{2}^{*}=-\beta_{1}^{r_{2}} x_{1}^{r_{2}}=-\beta_{1}^{r_{2}}\left[\xi_{1}\right]^{r_{2}}
$$

with $\beta_{1} \geq\left(n+\varphi_{1}\right)^{1 / r_{2} p_{1}}$ being smooth results in

$$
\dot{V}_{1} \leq-n\left|\xi_{1}\right|^{2}+\left[\xi_{1}\right]^{2-r_{2} p_{1}}\left(x_{2}^{p_{1}}-x_{2}^{* p_{1}}\right)
$$

Step $k(k=2, \ldots, n)$. Suppose at step $k-1$, there are a $C^{1}$, proper and positive definite Lyapunov function $V_{k-1}$, and a set of virtual controllers $x_{1}^{*}, \ldots, x_{k}^{*}$ defined by

$$
\begin{aligned}
x_{1}^{*}= & 0, \\
x_{2}^{*}= & -\beta_{1}^{r_{2}}\left[\xi_{1}\right]^{r_{2}}, \\
\vdots & \\
x_{k}^{*}= & -\beta_{k-1}^{r_{k}}\left[\xi_{k-1}\right]^{r_{k}}, \\
\xi_{1}= & {\left[x_{1}\right]^{1 / r_{1}}-\left[x_{1}^{*}\right]^{1 / r_{1}}, } \\
\xi_{2}= & {\left[x_{2}\right]^{1 / r_{2}}-\left[x_{2}^{*}\right]^{1 / r_{2}}, } \\
& \vdots \\
\xi_{k} & =\left[x_{k}\right]^{1 / r_{k}}-\left[x_{k}^{*}\right]^{1 / r_{k}},
\end{aligned}
$$

with $\beta_{1}>0, \ldots, \beta_{k-1}>0$ being smooth, such that

$$
\begin{array}{r}
\dot{V}_{k-1} \leq-(n-k+2) \sum_{j=1}^{k-1}\left|\xi_{j}\right|^{2}+\left[\xi_{k-1}\right]^{2-r_{k} p_{k-1}} \\
\times\left(x_{k}^{p_{k-1}}-x_{k}^{* p_{k-1}}\right) .
\end{array}
$$

To complete the induction, at the $k$ th step, we choose the following Lyapunov function:

$$
V_{k}\left(\bar{x}_{k}\right)=V_{k-1}\left(\bar{x}_{k-1}\right)+W_{k}\left(\bar{x}_{k}\right),
$$

where

$$
W_{k}=\int_{x_{k}^{*}}^{x_{k}}\left[[s]^{1 / r_{k}}-\left[x_{k}^{*}\right]^{1 / r_{k}}\right]^{2-r_{k+1} p_{k}} d s .
$$

Noting that $2-r_{k+1} p_{k} \geq 1$ and using a similar method as in [10], $V_{k}$ can be shown to be $C^{1}$, proper and positive definite. Moreover, we can obtain

$$
\begin{gathered}
\frac{\partial W_{k}}{\partial x_{k}}=\left[\xi_{k}\right]^{2-r_{k+1} p_{k}} \\
\frac{\partial W_{k}}{\partial x_{j}}=-\left(2-r_{k+1} p_{k}\right) \\
\times \int_{x_{k}^{*}}^{x_{k}}\left[[s]^{1 / r_{k}}-\left[x_{k}^{*}\right]^{1 / r_{k}}\right]^{1-r_{k+1} p_{k}} d s \frac{\partial\left[x_{k}^{*}\right]^{1 / r_{k}}}{\partial x_{j}},
\end{gathered}
$$

where $j=1, \ldots, k-1$.

Using (20)-(22), it follows that

$$
\begin{aligned}
\dot{V}_{k} \leq & -(n-k+2) \sum_{j=1}^{k-1}\left|\xi_{j}\right|^{2}+\left[\xi_{k-1}\right]^{2-r_{k} p_{k-1}}\left(x_{k}^{p_{k-1}}-x_{k}^{* p_{k-1}}\right) \\
& +\left[\xi_{k}\right]^{2-r_{k+1} p_{k}} x_{k+1}^{p_{k}}+\left[\xi_{k}\right]^{2-r_{k+1} p_{k}} f_{k}+\sum_{j=1}^{k-1} \frac{\partial W_{k}}{\partial x_{j}}\left(x_{j+1}^{p_{j}}+f_{j}\right) .
\end{aligned}
$$

In order to proceed further, an appropriate bounding estimate should be given for the last three terms on the righthand side of inequality (23). This is accomplished in the following propositions whose technical proofs are given in the appendix.

Proposition 7. There exists a positive constant $l_{k 1}$ such that

$$
\left[\xi_{k-1}\right]^{2-r_{k} p_{k-1}}\left(x_{k}^{p_{k-1}}-x_{k}^{* p_{k-1}}\right) \leq \frac{1}{3}\left|\xi_{k-1}\right|^{2}+l_{k 1}\left|\xi_{k}\right|^{2}
$$

Proposition 8. There exists a nonnegative smooth function $l_{k 2}$ such that

$$
\left[\xi_{k}\right]^{2-r_{k+1} p_{k}} f_{k} \leq \frac{1}{3} \sum_{j=1}^{k-1}\left|\xi_{j}\right|^{2}+l_{k 2}\left|\xi_{k}\right|^{2}
$$


Proposition 9. There exists a nonnegative smooth function $l_{k 3}$ such that

$$
\sum_{j=1}^{k-1} \frac{\partial W_{k}}{\partial x_{j}}\left(x_{j+1}^{p_{j}}+f_{j}\right) \leq \frac{1}{3} \sum_{j=1}^{k-1}\left|\xi_{j}\right|^{2}+l_{k 3}\left|\xi_{k}\right|^{2}
$$

Substituting (24)-(26) into (23) yields

$$
\begin{aligned}
\dot{V}_{k} \leq & -(n-k+1) \sum_{j=1}^{k-1}\left|\xi_{j}\right|^{2}+\left[\xi_{k}\right]^{2-r_{k+1} p_{k}} x_{k+1}^{p_{k}} \\
& +\left(l_{k 1}+l_{k 2}+l_{k 3}\right)\left|\xi_{k}\right|^{2} .
\end{aligned}
$$

Now, it easy to see that the virtual controller

$$
x_{k+1}^{*}=-\beta_{k}^{r_{k+1}}\left[\xi_{k}\right]^{r_{k+1}},
$$

where $\beta_{k} \geq\left(n-k+1+l_{k 1}+l_{k 2}+l_{k 3}\right)^{1 /\left(r_{k+1} p_{k}\right)}$ is a smooth function, renders

$$
\dot{V}_{k} \leq-(n-k+1) \sum_{j=1}^{k}\left|\xi_{j}\right|^{2}+\left[\xi_{k}\right]^{2-r_{k+1} p_{k}}\left(x_{k+1}^{p_{k}}-x_{k+1}^{* p_{k}}\right)
$$

\section{This completes the inductive step.}

Using the inductive argument above, we can conclude that at the $n$th step, there exists a continuous state feedback controller of the form

$$
u=x_{n+1}^{*}=-\beta_{n}^{r_{n+1}}\left[\xi_{n}\right]^{r_{n+1}}
$$

such that

$$
\dot{V}_{n} \leq-\sum_{j=1}^{n}\left|\xi_{j}\right|^{2}
$$

where

$$
V_{n}=\sum_{k=1}^{n} W_{k}=\sum_{k=1}^{n} \int_{x_{k}^{*}}^{x_{k}}\left[[s]^{1 / r_{k}}-\left[x_{k}^{*}\right]^{1 / r_{k}}\right]^{2-r_{k+1} p_{k}} d s .
$$

\section{Stability Analysis}

We state the main result in this paper.

Theorem 10. If Assumption 1 holds for system (1), under the continuous state feedback controller (30), then the following holds:

(i) all the solutions of the closed-loop system are well defined on $[0,+\infty)$;

(ii) the equilibrium $x=0$ of the closed-loop system is globally finite-time stable.

Proof. (i) Considering (18), system (1) can be equivalently transformed into a $\xi$-system:

$$
\dot{\xi}_{i}(t)=\varphi_{i}(t, \xi, u), \quad i=1, \ldots, n .
$$

By the existence and continuation of the solutions, states $\xi(t)$ are defined on $\left[0, t_{M}\right]$, where the number $t_{M}>0$ may be infinite or not. The following analysis focuses on $\left[0, t_{M}\right]$. Noting that $V_{n}$ is positive definite and radially unbounded, by (31), (32), and Lemma 4.3 in [24], it is not hard to obtain that there exist $K_{\infty}$ functions $\pi_{1}, \pi_{2}$, and $\pi_{3}$ such that

$$
\begin{gathered}
\pi_{1}(\|\xi(t)\|) \leq V_{n}(\xi(t)) \leq \pi_{2}(\|\xi(t)\|) \\
\dot{V}_{n} \leq-\pi_{3}(\|\xi(t)\|) .
\end{gathered}
$$

Since $\pi_{1}$ is a class $K_{\infty}$ function, then for any $\varepsilon>0$, one can always find a $\beta=\beta(\varepsilon)$ with $\beta>\varepsilon>0$ such that $\pi_{2}(\varepsilon) \leq \pi_{1}(\beta)$, $\forall t \in\left[0, t_{M}\right]$, which means that $\|\xi(t)\| \leq \beta, \forall t \in\left[0, t_{M}\right]$. Suppose that $t_{M}$ is finite; then $\lim _{t \rightarrow t_{M}}\|\xi(t)\|=+\infty$, which contradicts $\|\xi(t)\| \leq \beta, \forall t \in\left[0, t_{M}\right]$. Hence, $\xi(t)$ is well defined on $[0,+\infty)$, so is $x(t)$.

(ii) Noticing that $t_{M}=+\infty$, by using (34), and Lyapunov stability theorem [24], we know that the equilibrium $\xi=0$ of the closed-loop $\xi$-systems (30) and (33) is globally asymptotically stable. According to the definition of finite-time stability [11], if the global finitetime attractivity of the closed-loop system can be guaranteed, then the global finite-time stabilization result will be obtained. To this end, let us prove the global finite-time attractivity. First of all, by using Lemma 5 , it is easy to see that

$$
\begin{aligned}
W_{k} & =\int_{x_{k}^{*}}^{x_{k}}\left[[s]^{1 / r_{k}}-\left[x_{k}^{*}\right]^{1 / r_{k}}\right]^{2-r_{k+1} p_{k}} d s \\
& \leq\left|\xi_{k}\right|^{2-r_{k+1} p_{k}}\left|x_{k}-x_{k}^{*}\right| \leq 2^{1-r_{k}}\left|\xi_{k}\right|^{2-\omega} .
\end{aligned}
$$

So we have the following estimate:

$$
V_{n}=\sum_{k=1}^{n} W_{k} \leq 2 \sum_{k=1}^{n}\left|\xi_{k}\right|^{2-\omega}
$$

Let $\alpha=2 /(2-\omega)$. With (36) and (31) in mind, by Lemma 4 , it is not difficult to obtain that

$$
\dot{V}_{n} \leq-\frac{1}{2} V_{n}^{\alpha}
$$

Therefore, by Lemma 3, we obtain that the equilibrium $\xi=0$ of the closed-loop $\xi$-systems (30) and (33) is globally finite-time stable. This together with the definitions of $x_{i}^{*}$ s directly concludes that the globally finite-time stability of the closed-loop systems (1), (18), and (30) at the equilibrium $x=$ 0 .

\section{Simulation Example}

To illustrate the effectiveness of the proposed approach, we consider the following low-dimensional system:

$$
\begin{aligned}
& \dot{x}_{1}=x_{2}^{5 / 3}+x_{1}^{10 / 11}, \\
& \dot{x}_{2}=u+x_{1}^{2} \sin x_{2},
\end{aligned}
$$




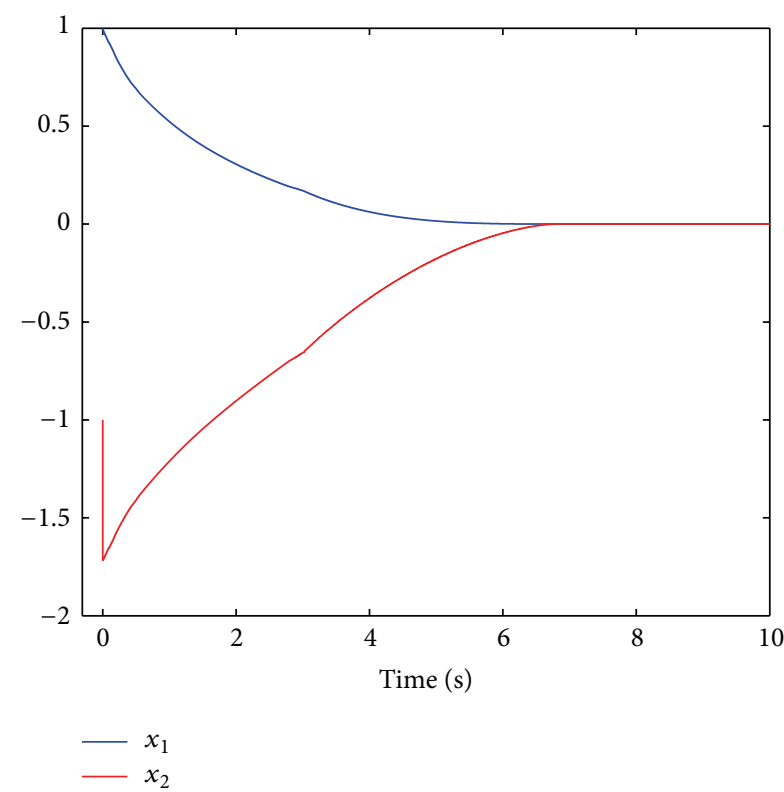

(a) System states

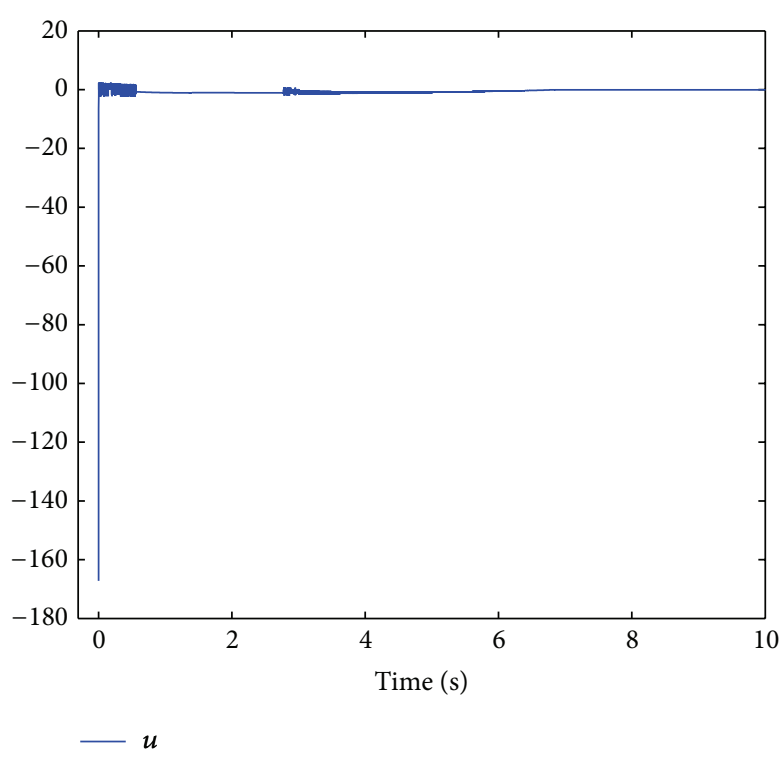

(b) Control input

FIGURE 1: The responses of the closed-loop system (38) and (39).

where $p_{1}=5 / 3$ and $p_{2}=1$. It is worth pointing out that although system (38) is simple, it cannot be globally finite-time stabilized using the design method presented in $[14,21,22]$ because of the presence of both low-order term $x_{1}^{10 / 11}$ and high-order term $x_{1}^{2} \sin x_{2}$. Choose $\omega=-1 / 11 \in(-3 / 8,0]$; then $r_{1}=1, r_{2}=\left(r_{1}+\omega\right) / p_{1}=6 / 11$, and $r_{3}=\left(r_{2}+\omega\right) / p_{2}=5 / 11$. By Lemma 6 , it is easy to get $\left|f_{1}\right| \leq\left|x_{1}\right|^{10 / 11}$ and $\left|f_{2}\right| \leq\left(1+x_{1}^{2}\right)\left|x_{1}\right|^{5 / 11}$. Clearly, Assumption 1 holds with $\varphi_{1}=1$ and $\varphi_{2}=1+x_{1}^{2}$; according to the design procedure proposed in Section 3, we can get

$$
\begin{gathered}
x_{2}^{*}=-\beta_{1}^{6 / 11}\left[x_{1}\right]^{6 / 11}, \quad \beta_{1}=3^{11 / 10}, \\
u=-\left(1+l_{21}+l_{22}+l_{23}\right)\left[\xi_{2}\right]^{5 / 11},
\end{gathered}
$$

where $\xi_{2}=\left[x_{2}\right]^{11 / 6}-\left[x_{2}^{*}\right]^{11 / 6}, l_{21}=(5 / 11) \cdot(18 / 11)^{6 / 5} \cdot 2^{1 / 5}$, $l_{22}=(17 / 22) \cdot(15 / 22)^{5 / 17} \cdot\left(1+x_{1}^{2}\right)^{22 / 17}$, and $l_{23}=(6 /$ $11) \cdot(30 / 11)^{5 / 6} \cdot(17 / 11)^{11 / 6} \cdot\left(3^{121 / 60}+3^{121 / 30}\right)$.

In the simulation, by choosing the initial values as $x_{1}(0)=1$ and $x_{2}(0)=-1$, Figure 1 is obtained to demonstrate the effectiveness of the control scheme.

\section{Conclusion}

In this paper, a continuous state feedback stabilizing controller is presented for a class of high-order nonlinear systems under weaker condition. The controller designed preserves the equilibrium at the origin and guarantees the global finite-time stability of the systems. It should be noted that the proposed controller can only work well when the whole state vector is measurable. Therefore, a natural and more interesting problem is how to design output feedback stabilizing controller for the systems studied in the paper if only partial state vector being measurable, which is now under our further investigation.

\section{Appendix}

Proof of Proposition 7. Noting that $-1 / \sum_{l=1}^{n} p_{1} \cdots p_{l-1}<\omega<$ 0 and $p_{k-1} r_{k}=r_{k-1}+\omega$, we have $0<p_{k-1} r_{k}<1$. Using (18), it follows from Lemma 5 that

$$
\begin{aligned}
\left|x_{k}^{p_{k-1}}-x_{k}^{* p_{k-1}}\right| & =\left|\left(x_{k}^{1 / r_{k}}\right)^{r_{k} p_{k-1}}-\left(x_{k}^{*\left(1 / r_{k}\right)}\right)^{r_{k} p_{k-1}}\right| \\
& \leq 2^{1-r_{k} p_{k-1}}\left|\left[x_{k}\right]^{1 / r_{k}}-\left[x_{k}^{*}\right]^{1 / r_{k}}\right|^{r_{k} p_{k-1}} \\
& =2^{1-r_{k} p_{k-1}}\left|\xi_{k}\right|^{r_{k} p_{k-1}} .
\end{aligned}
$$

By (A.1) and Lemma 6, it can be obtained that

$$
\begin{aligned}
& {\left[\xi_{k-1}\right]^{2-r_{k} p_{k-1}}\left(x_{k}^{p_{k-1}}-x_{k}^{* p_{k-1}}\right)} \\
& \quad \leq 2^{1-r_{k} p_{k-1}}\left|\xi_{k-1}\right|^{2-r_{k} p_{k-1}}\left|\xi_{k}\right|^{r_{k} p_{k-1}} \\
& \quad \leq \frac{1}{3}\left|\xi_{k-1}\right|^{2}+l_{k 1}\left|\xi_{k}\right|^{2},
\end{aligned}
$$

where $l_{k 1}>0$ is a constant.

Proof of Proposition 8. According to (18), Assumption 1, and Lemma 4, it follows that

$$
\begin{aligned}
\left|f_{i}\right| & \leq \varphi_{i} \sum_{j=1}^{i}\left|x_{j}(t)\right|^{\left(r_{i}+\omega\right) / r_{j}} \\
& \leq \varphi_{i} \sum_{j=1}^{i}\left(\left|\xi_{j}\right|+\beta_{j-1}\left|\xi_{j-1}\right|\right)^{r_{i+1} p_{i}}
\end{aligned}
$$




$$
\begin{aligned}
& \leq 2^{1-r_{i+1} p_{i}} \varphi_{i} \sum_{j=1}^{i}\left(\left|\xi_{j}\right|^{r_{i+1} p_{i}}+\beta_{j-1}^{r_{i+1} p_{i}}\left|\xi_{j-1}\right|^{r_{i+1} p_{i}}\right) \\
& \leq \bar{\varphi}_{i} \sum_{j=1}^{i}\left|\xi_{j}\right|^{r_{i+1} p_{i}},
\end{aligned}
$$

where $\beta_{0}=\xi_{0}=0$ and $\bar{\varphi}_{i}=2^{1-r_{i+1} p_{i}} \sum_{j=1}^{i}\left(1+\beta_{j-1}^{r_{i+1}} p_{i}\right) \varphi_{i} \geq 0$ is a smooth function.

Using (A.3) and Lemmas 5 and 6, we obtain

$$
\begin{aligned}
{\left[\xi_{k}\right]^{2-r_{k+1} p_{k}} f_{k} } & \leq \bar{\varphi}_{k} \sum_{j=1}^{k}\left|\xi_{k}\right|^{2-r_{k+1} p_{k}}\left|\xi_{j}\right|^{r_{k+1} p_{k}} \\
& \leq \frac{1}{3} \sum_{j=1}^{k-1}\left|\xi_{j}\right|^{2}+l_{k 2}\left|\xi_{k}\right|^{2}
\end{aligned}
$$

where $l_{k 2} \geq 0$ is a smooth function.

Proof of Proposition 9. Note that

$$
\left[x_{j+1}^{*}\right]^{1 /\left(r_{j+1}\right)}=-\beta_{j} \xi_{j}=-\sum_{j=1}^{k-1} B_{j}\left[x_{j}\right]^{1 / r_{j}}
$$

where $B_{j}=\beta_{k-1} \cdots \beta_{j}$ and $j=1, \ldots, k-1$.

Using (A.5), after simple calculations, it is not hard to obtain that for $j=1, \ldots, k-1$,

$$
\frac{\partial\left[x_{k}^{*}\right]^{1 / r_{k}}}{\partial x_{j}}=-\sum_{l=1}^{k-1} \frac{\partial B_{l}}{\partial x_{j}}\left[x_{j}\right]^{1 / r_{j}}-\frac{1}{r_{j}} B_{j}\left|x_{j}\right|^{1 / r_{j}-1} .
$$

By (22), (A.3), (A.6), and Lemmas 4 and 5, we get

$$
\begin{aligned}
\sum_{j=1}^{k-1} \frac{\partial W_{k}}{\partial x_{j}}\left(x_{j+1}^{p_{j}}+f_{j}\right) & -\left(2-r_{k+1} p_{k}\right) \int_{x_{k}^{*}}^{x_{k}}\left[[s]^{1 / r_{k}}-\left[x_{k}^{*}\right]^{1 / r_{k}}\right]^{1-r_{k+1} p_{k}} d s \\
= & \times \sum_{j=1}^{k-1} \frac{\partial\left[x_{k}^{*}\right]^{1 / r_{k}}}{\partial x_{j}}\left(x_{j+1}^{p_{j}}+f_{j}\right) \\
\leq & 2^{2-r_{k}}\left|\xi_{k}\right|^{1-\omega} \sum_{j=1}^{k-1}\left|\frac{\partial\left[\alpha_{k-1}\right]^{1 / r_{k}}}{\partial x_{j}}\right|\left(\left|x_{j+1}\right|^{p_{j}}+\left|f_{j}\right|\right) \\
\leq & 2^{2-r_{k}}\left|\xi_{k}\right|^{1-\omega} \sum_{j=1}^{k-1}\left|\sum_{l=1}^{k-1} \frac{\partial B_{l}}{\partial x_{j}}\right| x_{j}\left|+\frac{1}{r_{j}} B_{j}\right|\left|x_{j}\right|^{1 / r_{j}-1} \\
& \times\left(\left|x_{j+1}\right|^{p_{j}}+\left|f_{j}\right|\right) \\
\leq & \sum_{j=1}^{k-1} \bar{B}_{j}\left|\xi_{k}\right|^{1-\omega}\left|x_{j}\right|^{1 / r_{j}-1}\left(\left|x_{j+1}\right| p_{j}+\left|f_{j}\right|\right)
\end{aligned}
$$

$$
\begin{aligned}
\leq & \sum_{j=1}^{k-1} \bar{B}_{j}\left|\xi_{k}\right|^{1-\omega}\left|\xi_{j}+\beta_{j-1} \xi_{j-1}\right|^{1-r_{j}} \\
& \times\left(\left|\xi_{j+1}+\beta_{j} \xi_{j}\right|^{r_{j+1} p_{j}}+\bar{\varphi}_{i} \sum_{l=1}^{j}\left|\xi_{l}\right|^{r_{j+1} p_{j}}\right),
\end{aligned}
$$

where $\bar{B}_{j} \geq 0$ is a smooth function.

Noting that $r_{j+1} p_{j}=r_{j}+\omega$, by using Lemma 6, we have

$$
\sum_{j=1}^{k-1} \frac{\partial W_{k}}{\partial x_{j}}\left(x_{j+1}^{p_{j}}+f_{j}\right) \leq \frac{1}{3} \sum_{j=1}^{k-1}\left|\xi_{j}\right|^{2}+l_{k 3}\left|\xi_{k}\right|^{2},
$$

where $l_{k 3} \geq 0$ is a smooth function.

\section{Conflict of Interests}

The authors declare that there is no conflict of interests regarding the publication of this paper.

\section{Acknowledgments}

The authors thank the editor and the anonymous reviewers for their constructive comments and suggestions for improving the quality of the paper. This work has been supported in part by the National Nature Science Foundation of China under Grant 61073065 and the Key Program of Science Technology Research of Education Department of Henan Province under Grants 13A120016 and 14A520003.

\section{References}

[1] C. Rui, M. Reyhanoglu, I. Kolmanovsky, S. Cho, and N.H. McClamroch, "Nonsmooth stabilization of an underactuated unstable two degrees of freedom mechanical system," in Proceedings of the 36th IEEE Conference Decision Control, vol. 4, pp. 3998-4003, 1997.

[2] W. Lin and C. Qian, "Adding one power integrator: a tool for global stabilization of high-order lower-triangular systems," Systems \& Control Letters, vol. 39, no. 5, pp. 339-351, 2000.

[3] C. Qian and W. Lin, "A continuous feedback approach to global strong stabilization of nonlinear systems," IEEE Transactions on Automatic Control, vol. 46, no. 7, pp. 1061-1079, 2001.

[4] W. Lin and C. Qian, "Adaptive control of nonlinearly parameterized systems: a nonsmooth feedback framework," IEEE Transactions on Automatic Control, vol. 47, no. 5, pp. 757-774, 2002.

[5] W. Lin and C. Qian, "Adaptive control of nonlinearly parameterized systems: the smooth feedback case," IEEE Transactions on Automatic Control, vol. 47, no. 8, pp. 1249-1266, 2002.

[6] J. Polendo and C. Qian, "A generalized homogeneous domination approach for global stabilization of inherently nonlinear systems via output feedback," International Journal of Robust and Nonlinear Control, vol. 17, no. 7, pp. 605-629, 2007.

[7] J. Polendo and C. Qian, "An expanded method to robustly stabilize uncertain nonlinear systems," Communications in Information and Systems, vol. 8, no. 1, pp. 55-70, 2008. 
[8] Z. Sun and Y. Liu, "Adaptive stabilisation for a large class of high-order uncertain non-linear systems," International Journal of Control, vol. 82, no. 7, pp. 1275-1287, 2009.

[9] J. Zhang and Y. Liu, "A new approach to adaptive control design without overparametrization for a class of uncertain nonlinear systems," Science China: Information Sciences, vol. 54, no. 7, pp. 1419-1429, 2011.

[10] X.-H. Zhang and X.-J. Xie, "Global state feedback stabilisation of nonlinear systems with high-order and low-order nonlinearities," International Journal of Control, vol. 87, no. 3, pp. 642-652, 2014.

[11] S. P. Bhat and D. S. Bernstein, "Finite-time stability of continuous autonomous systems," SIAM Journal on Control and Optimization, vol. 38, no. 3, pp. 751-766, 2000.

[12] Y. Hong, J. Huang, and Y. Xu, "On an output feedback finitetime stabilization problem," IEEE Transactions on Automatic Control, vol. 46, no. 2, pp. 305-309, 2001.

[13] Y. Hong, "Finite-time stabilization and stabilizability of a class of controllable systems," Systems \& Control Letters, vol. 46, no. 4, pp. 231-236, 2002.

[14] X. Huang, W. Lin, and B. Yang, "Global finite-time stabilization of a class of uncertain nonlinear systems," Automatica, vol. 41, no. 5, pp. 881-888, 2005.

[15] Y. Hong, J. Wang, and D. Cheng, "Adaptive finite-time control of nonlinear systems with parametric uncertainty," IEEE Transactions on Automatic Control, vol. 51, no. 5, pp. 858-862, 2006.

[16] Y. Hong and Z.-P. Jiang, "Finite-time stabilization of nonlinear systems with parametric and dynamic uncertainties," IEEE Transactions on Automatic Control, vol. 51, no. 12, pp. 1950-1956, 2006.

[17] S. Li and Y.-P. Tian, "Finite-time stability of cascaded timevarying systems," International Journal of Control, vol. 80, no. 4, pp. 646-657, 2007.

[18] S. G. Nersesov, W. M. Haddad, and Q. Hui, "Finite-time stabilization of nonlinear dynamical systems via control vector Lyapunov functions," Journal of the Franklin Institute: Engineering and Applied Mathematics, vol. 345, no. 7, pp. 819-837, 2008.

[19] H. Du, C. Qian, M. T. Frye, and S. Li, "Global finite-time stabilisation using bounded feedback for a class of non-linear systems," IET Control Theory \& Applications, vol. 6, no. 14, pp. 2326-2336, 2012.

[20] S. Ding, S. Li, and W. X. Zheng, "Nonsmooth stabilization of a class of nonlinear cascaded systems," Automatica, vol. 48, no. 10, pp. 2597-2606, 2012.

[21] Y. Shen and Y. Huang, "Global finite-time stabilisation for a class of nonlinear systems," International Journal of Systems Science: Principles and Applications of Systems and Integration, vol. 43, no. 1, pp. 73-78, 2012.

[22] J. Li, C. Qian, and S. Ding, "Global finite-time stabilisation by output feedback for a class of uncertain nonlinear systems," International Journal of Control, vol. 83, no. 11, pp. 2241-2252, 2010.

[23] B. Yang and W. Lin, "Nonsmooth output feedback design with a dynamics gain for uncertain systems with strong nonlinearities," in Proceedings of the 46th IEEE Conference Decision Control, pp. 3495-3500, New Orieans, La, USA, 2007.

[24] H. K. Khalil, Nonlinear Systems, Prentice-Hall, New Jersey, NJ, USA, 3rd edition, 2002. 


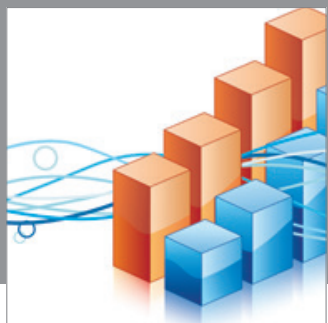

Advances in

Operations Research

mansans

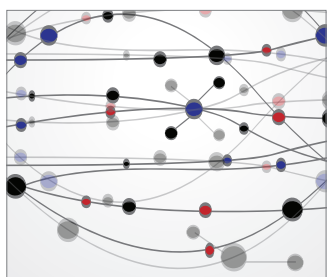

The Scientific World Journal
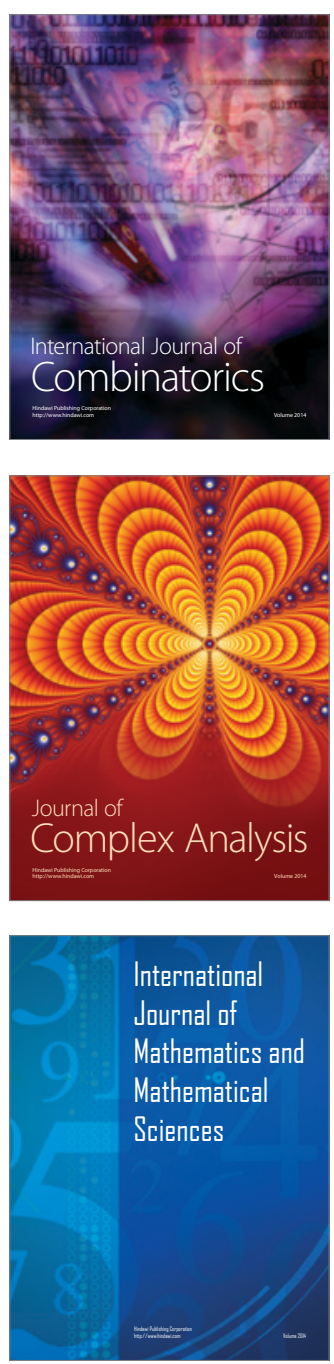
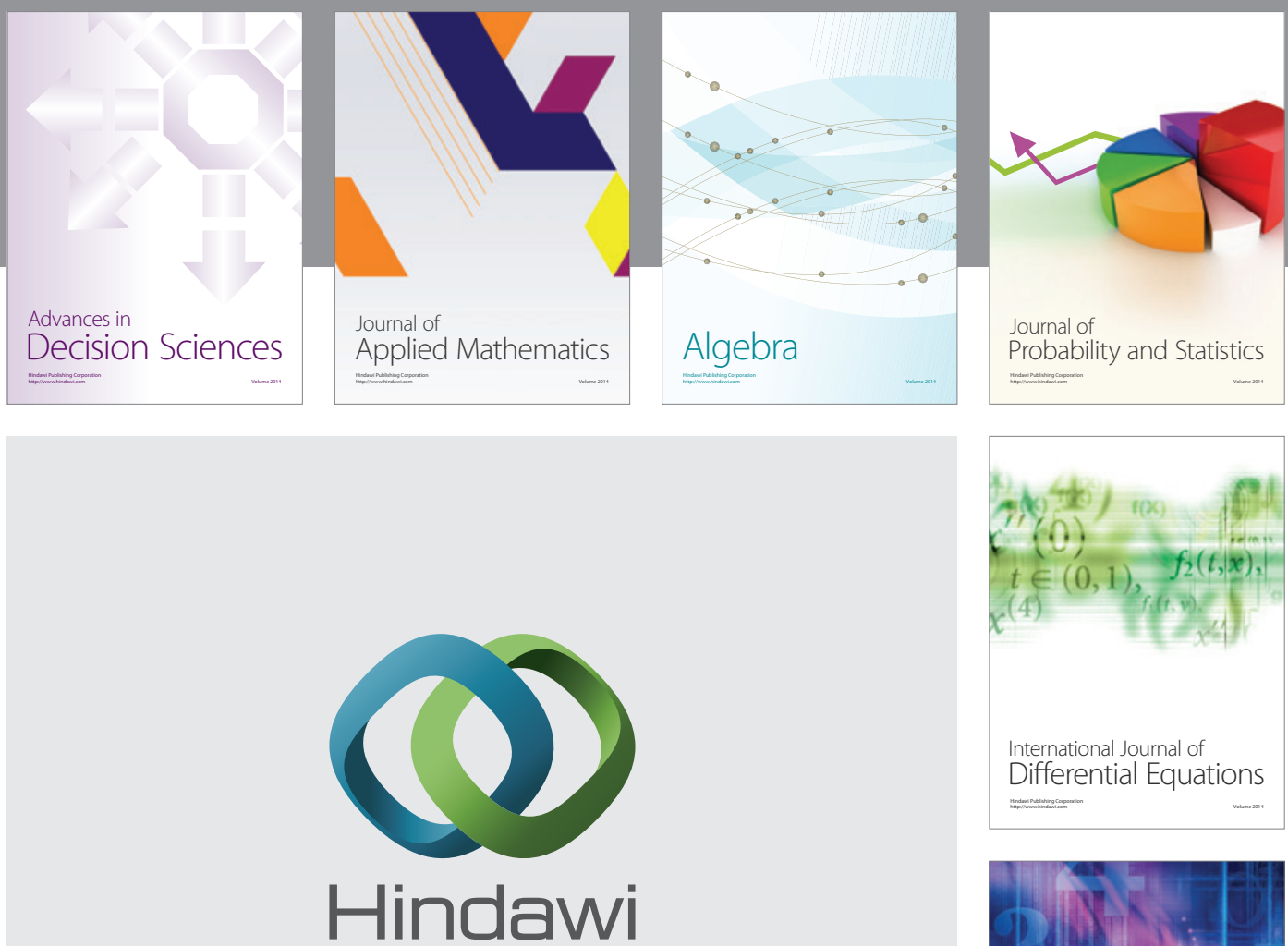

Submit your manuscripts at http://www.hindawi.com
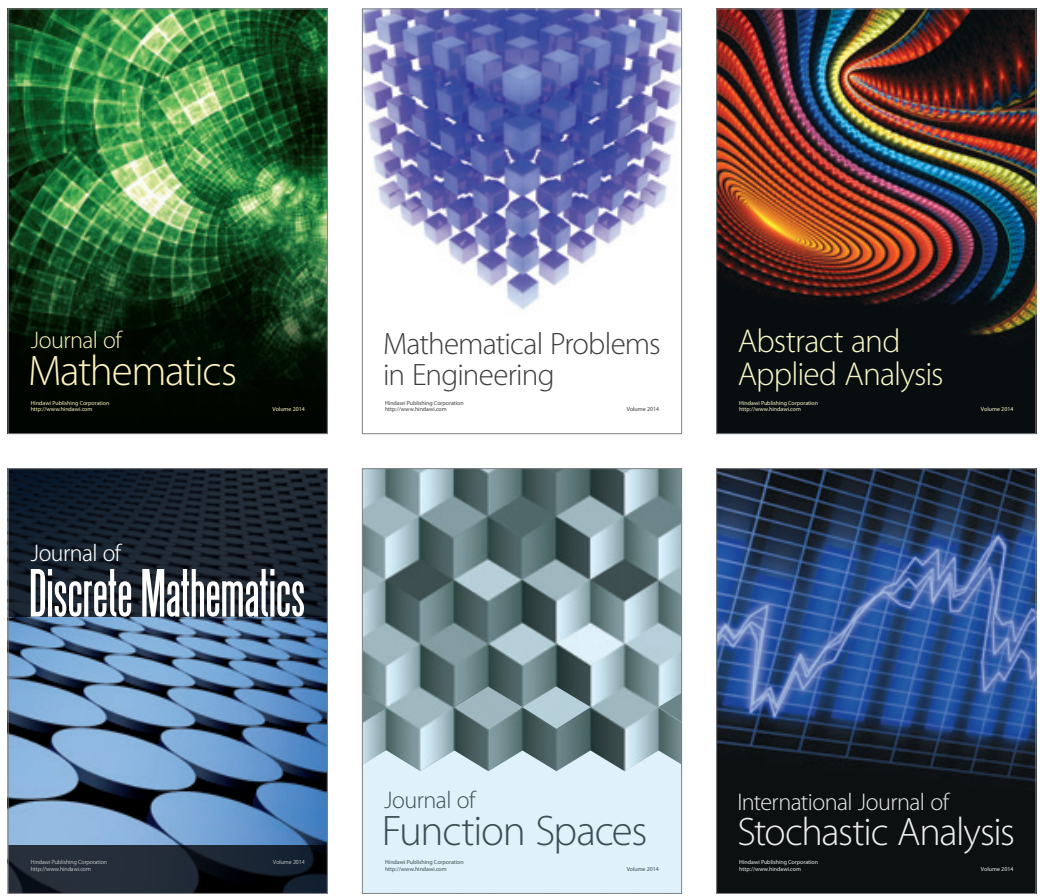

Journal of

Function Spaces

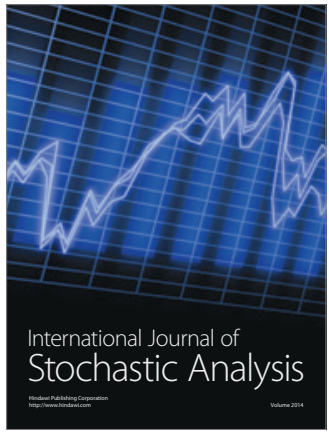

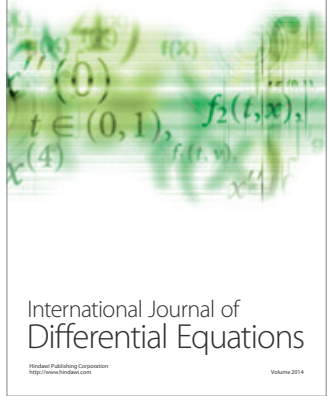
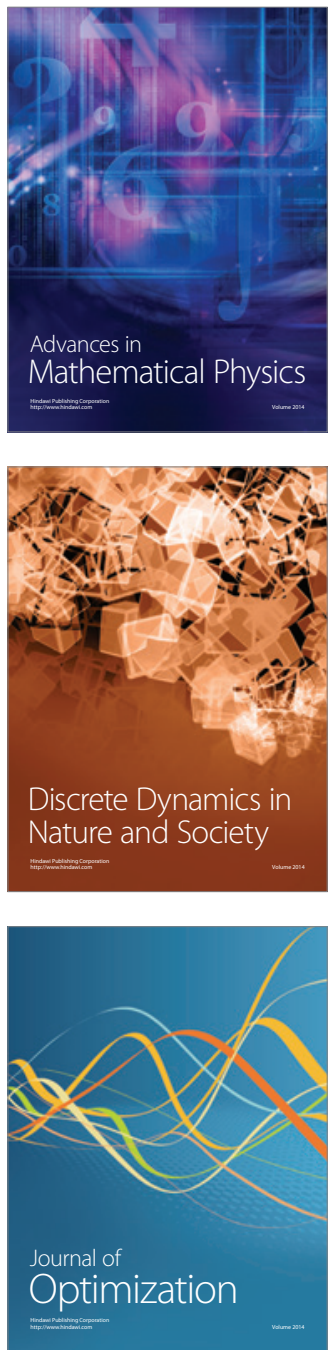